\title{
Discrepancy between food offered and consumed during hospitalization in older adult patients
}

\section{Abstract}

Malnutrition is one of the most frequent geriatric syndromes and a key contributor to frailty. Ageing is commonly associated with modifications in eating habits with changes in appetite and food intake. Malnutrition is not only a sign of illness, but its presence increases morbidity, hospital stay, institutionalization, poor quality of life, in-hospital complications, expenses, and mortality. Hospitalized patients have increased calorie requirements to overcome the increased energy expenditure due to acute disease. We aimed to evaluate the actual consumption of the food offered to a sample of hospitalized older adults. Sixty hospitalized patients (women/men $=23 / 37$ ) were recruited sequentially. After participants signed an informed consent, we evaluated their mean actual intake of macronutrients (carbohydrates, proteins, and fats) at lunch during three consecutive days, calculated with a nutrition analysis software from the weighted food (first course, second course, side dishes) measured before and after consumption. Other parameters assessed included self-sufficiency by the capacity to perform basic and instrumental activities of daily living (ADL, IADL), cognitive performance with Mini Mental State Evaluation (MMSE), Geriatric Depression Scale (GDS), physical performance with the Short Physical Performance Battery (SPPB) and handgrip dynamometry, EAT-10 test for the assessment of swallowing capacity, and anthropometric parameters. Mean \pm SD of the main parameters assessed were as follows: age $77.8 \pm 9.3$ years, BMI $27.1 \pm 8.7 \mathrm{Kg} / \mathrm{m}^{2}$, ADL $3.8 \pm 2.0$, IADL $4.1 \pm 2.8$ (indicative of compromise self-efficacy), MMSE $21.8 \pm 5.9$, SSPB 5.3 \pm 3.1, handgrip dynamometry $16.5 \pm 7.8 \mathrm{Kg}$ (indicative of mild to moderate cognitive and physical impairment). The mean intake of macronutrients for the 3 -day assessment was $65.7 \pm 20.6 \%$ of carbohydrates, $74.3 \pm 30.4 \%$ of proteins, and $47.4 \pm 38.1 \%$ of fats of the total amount offered at lunch. Older patients tended to have lower intakes of carbohydrates, proteins, and fats, while participants with higher SPPB had higher intakes of carbohydrates and proteins. The results of the present study show that hospitalized older adults, with compromised autonomy, physical and cognitive performance, had lower consumption compared to what is offered to them in the hospital despite their elevated nutritional needs due to the acute illness leading to hospitalization. Undernutrition and weight-loss are key factors associated with increased mortality and morbidity. Therefore, it is crucial to set appropriate nutritional interventions in hospitalized patients, particularly those with disability and cognitive decline. Early recognition and treatment of malnutrition are essential preventive measures to improve the quality of care, quality of life, and decrease mortality risk in hospitalized older adults.

\section{Conflict of Interest}

There is no conflict of interest 